\title{
Concentraciones de homocisteína en plasma en pacientes españoles con enfermedad arterial coronaria
}

\author{
A. DOMÍNGUEZ RODRÍGUEZ, P. ABREU GONZÁLEZ*, A. JIMENEZ SOSA**, \\ M. J. GARCÍA GONZÁLEZ, A. BARRAGÁN ACEA, D. DE ARMAS TRUJILLO †
}

Servicio de Cardiología del Hospital Universitario de Canarias. *Departamento de Fisiología, Universidad de La Laguna. **Unidad de Investigación del Hospital Universitario de Canarias.

\author{
PLASMA HOMOCYSTEINE LEVELS IN SPANISH PATIENTS WITH \\ CORONARY ARTERY DISEASE
}

\section{RESUMEN}

Objetivo: El aumento de las concentraciones séricas del aminoácido homocisteína (HCY) es un posible factor de riesgo de enfermedades cardiovasculares. Los mecanismos propuestos para explicar porqué la $\mathrm{HCY}$ puede hacer aumentar el riesgo de enfermedades vasculares son un efecto directo sobre el endotelio vascular y su intervención en el aumento del riesgo de trombosis. El presente estudio ha sido diseñado para conocer la homocisteinemia de pacientes residentes en las Islas Canarias con enfermedad arterial coronaria (EAC), y comprobar si la hiperhomocisteinemia es un factor de riesgo.

Métodos: Se incluyeron 132 pacientes afectos de EAC demostrada angiográficamente y 18 controles con arterias coronarias normales. Se determinaron los valores de $\mathrm{HCY}$, vitamina B12, vitamina B6, ácido fólico, creatinina, colesterol con sus diferentes fracciones, triglicéridos, glucosa y fibrinógeno.

Resultados: Las concentraciones medias de HCY no difirieron significativamente $(\mathrm{p}=0,37)$ entre los casos y los sujetos de control. La distribución de los niveles de HCY por quintiles no parece estar asociada sobre la producción de EAC $(\mathrm{p}=0,57)$. El análisis de regresión logística múltiple de los factores de riesgos comparado con los quintiles 2, 3, 4 y 5 de HCY no demostró evidencia significativa entre la concentración de HCY y el riesgo de EAC.

Conclusiones: Este estudio añade incertidumbre de que la hiperhomocisteinemia sea factor de riesgo para EAC. Es necesario realizar estudios controlados de intervención clínica, algunos de los cuales están en marcha, para intentar aclarar las interrelaciones entre la HCY total y las enfermedades cardiovasculares.

PALABRAS CLAVE: Enfermedad Coronaria. Enfermedades cardiovasculares. Cateterismo cardíaco.

\section{ABSTRACT \\ Objective: A rise in plasma levels of the amino acid homocysteine} $(\mathrm{HCY})$ is a possible risk factor in cardiovascular disease. The mechanisms proposed to explain how HCY can increase the risk of vascular disease include its direct effect on the vascular endothelium and its role in increasing the risk of thrombosis. The present work has been designed to determine HCY levels in patients with coronary artery disease (CAD) residents in the Canary Islands and to establish whether hyperhomocys teinemia can be considered as an risk factor.

Methods: The sample studied consisted of 132 patients with, angio graphically demonstrated, CAD and 18 controls with normal coronary arteries. Biochemical parameters determined included: $H C Y$, vitamin B12, vitamin B6, folic acid, creatinine, cholesterol and its fractions, triglycerides, glucose and fibrinogen.

Results: Mean levels of $\mathrm{HCY}$ were not significantly different between the cases and controls $(p=0.37)$. In the distribution of $\mathrm{HCY}$ levels into quintiles there was no significant association between the quintiles and the occurrence of CAD $(p=0.57)$. Multiple logistic regression analysis in which the risk factors were compared with quintiles 2,3,4 and 5 of $\mathrm{HCY}$ did not reveal a significant relation between $\mathrm{HCY}$ levels and risk of CAD.

Conclusions: This study questions the previously accepted conside ration that hyperhomocysteinemia is a risk factor of CAD. Controlled intervention trials are, therefore, necessary to clarify the possible asso ciation between total HCY levels and cardiovascular disease.

KEY WORDS: Coronay Disease. Cardiovascular diseases. Catheteriza tion.

Domínguez Rodríguez A, Abreu González P, Jimenez Sosa A, García González MJ, Barragán Acea A, de Armas Trujillo D. Concentraciones de homocisteína en plasma en pacientes españoles con enfermedad arterial coronaria. An Med Interna (Madrid) 2002; 19: 166-170.

\section{INTRODUCCIÓN}

La HCY es un aminoácido sulfurado que se produce en los vertebrados a partir de la metionina. Las concentraciones de HCY plasmática pueden elevarse por defectos genéticos en las enzimas implicadas en su metabolismo o por deficiencias nutricionales en los cofactores vitamíni$\cos (1)$. La homocistinuria es un raro déficit autosómico recesivo de la cistationina $b$ sintetasa que se asocia a una elevación de la concentración de HCY total en sangre con una incidencia muy elevada de enfermedades vasculares prematuras.

$\dagger$ † título póstumo D.E.P

Trabajo aceptado: 14 de enero de 2002

Correspondencia: Alberto Domínguez Rodríguez. Hospital Universitario de Canarias. Ctra. La Cuesta-Taco, s/n. 38320 Ofra. La Laguna. Santa Cruz de Tenerife. 
Se cree que la elevación moderada de la HCY es un factor de riesgo de EAC, ictus aterotrombótico y enfermedad vascular periférica. De once estudios prospectivos publicados, sólo seis de ellos han encontrado que la concentración de HCY total sea un factor de riesgo de enfermedad vascular ateromatosa (2), inclusive en uno de estos estudios (3), cuando se amplió a 2,5 años, dejó de mostrar la asociación estadísticamente significativa entre HCY total e incidencia de EAC (4), no encontrando posteriormente una relación entre HCY total y riesgo de angina de pecho (5). En los pacientes con EAC, la hiperhomocisteinemia varía entre el $9 \%$ (6) y el $49 \%$ (7) de los casos. Se suele utilizar el criterio de considerar hiperhomocisteinémicos a las personas con concentraciones plasmáticas de HCY basal y/o con incrementos postmetionina que son superiores a la media más dos desviaciones estándar de los controles o, alternativamente, aquellos con concentraciones superiores al percentil 90 o $95 \%$ de éstos (8). Arbitrariamente se clasifica la hiperhomocisteinemia en moderada (16-30 $\mu \mathrm{mol} / \mathrm{L})$, intermedia (31-100 $\mu \mathrm{mol} / \mathrm{L})$ y severa (> $100 \mu \mathrm{mol} / \mathrm{L})$. En un estudio español (9) se ha comunicado que en el $26 \%$ de los pacientes con EAC se comprueba hiperhomocisteinemia.

El presente estudio ha sido diseñado para conocer la homocisteinemia de pacientes residentes en las Islas Canarias con EAC, y comprobar si la hiperhomocisteinemia es factor de riesgo.

\section{PACIENTES Y MÉTODOS}

Pacientes: entre mayo de 1999 y abril de 2000 fueron atendidos 614 pacientes en la Unidad Coronaria del Hospital Universitario de Canarias. De estos, 132 pacientes se incluyeron en el presente estudio por cumplir los siguientes criterios de inclusión: existencia de EAC, ausencia de tratamiento multivitamínico o anticonvulsivante y de enfermedad sistémica aguda o maligna. La EAC se definió como antecedente de infarto de miocardio (IM) previo mayor o igual a 6 meses, sindrome anginoso o demostración por angiografía coronaria de lesiones ateromatosas en una o más arterias principales coronarias. En lo que se refiere a la raza se controló, introduciendo entre los criterios de inclusión sólo sujetos blancos. Del mismo modo se controló la diabetes, introduciendo entre los criterios de inclusión sólo sujetos no diabéticos. La angiografía coronaria se realizó en los 132 pacientes. Los controles fueron 18 sujetos ingresados de forma programada para la realización de cateterismo cardíaco con el diagnóstico de valvulopatías severas del tipo de insuficiencia mitral y estenosis aórtica, demostrándose por angiografía coronaria la existencia de arterias coronarias normales. Entre los criterios de exclusión de los pacientes en el estudio tenemos: función renal con creatinina mayor de $1,7 \mathrm{mg} / \mathrm{dl}$, enfermedades hepáticas, patología tiroidea, embarazo, enfermedades psiquiátricas y exposición a óxido nitroso.

Las variables controladas fueron: sexo, edad, tabaquismo, hipertensión arterial, hipercolesterolemia, hipertrigliceridemia e índice de masa corporal.

Determinaciones de laboratorio: todas las muestras de sangre se extrajeron con un ayuno de al menos 12 horas. Se determinaron los valores plasmáticos de $\mathrm{HCY}$, vitamina $\mathrm{B}_{12}$, vitamina $\mathrm{B}_{6}$, ácido fólico, colesterol total y sus diferentes fracciones, triglicéridos, creatinina, fibrinógeno y glucosa.

La sangre recogida en $\mathrm{K}_{2}$ EDTA se colocó inmediatamente en hielo y se centrifugó dentro de la hora posterior a la extrac- ción. Una vez separado el plasma, se congeló a $-20^{\circ} \mathrm{C}$ hasta su análisis. Los valores de HCY plasmática se determinaron por medio de un inmunoensayo enzimático adaptado a microplacas (10). La HCY total de la muestra es determinada como la S-adenosil-L-HCY en un inmunoensayo competitivo con un anticuerpo monoclonal contra la S-adenosil-L-HCY. La vitamina $B_{12}$ y el ácido fólico se determinaron por radioinmunoanálisis de doble marcaje ( ${ }^{125} \mathrm{I}$ y ${ }^{57} \mathrm{Co}$ ) (DRG Instruments Gmbh, Alemania). La vitamina $B_{6}$ se determinó por Radioinmunoanálisis (Centro Inmunológico de Cataluña, España). Los niveles de glucosa, triglicéridos, creatinina y colesterol total con sus diferentes fracciones se determinaron por un test enzimático colorimétrico. El fibrinógeno se determinó por un test turbidimétrico.

Análisis estadístico: en un estudio previo (11) se obtuvieron los siguientes valores promedios de HCY en una cohorte de pacientes con EAC y un grupo control: $8,86 \mu \mathrm{mol} / \mathrm{L}$ y $8,53 \mu \mathrm{mol} / \mathrm{L}$, respectivamente. Para demostrar diferencias entre los promedios mencionados, con desviación estandar de $0,8 \mu \mathrm{mol} / \mathrm{L}$, se requieren 132 pacientes con EAC. La potencia se fija en el $86 \%$ y el error tipo I en 5\% (12) . Los resultados de las variables cuantitativas se expresan como medias y desviaciones estándar; y las variables categóricas en porcentajes. Se utilizó el Test de Levene para comprobar la asunción de normalidad de las distribuciones de frecuencias de la HCY en los pacientes y los controles. Se realizó una transformación logarítmica de los valores de HCY y se categorizaron en quintiles. Se utilizó el análisis de covarianza (ANCOVA) para comparar los promedios de HCY entre pacientes y controles, mientras se controlaba por edad; sexo; presencia de tabaquismo; hipertensión arterial y colesterol. Para expresar clínicamente la magnitud de la asociación entre niveles de HCY y ser clasificado como paciente (lesión en arteria coronaria), se realizó un análisis de regresión logística incondicional con método de selección de variables paso a paso hacia delante. El resultado se expresa con la Odds Ratio y su intervalo de confianza al 95\%. Se consideró significativo un valor de $\mathrm{p}<0,05$.

\section{RESULTADOS}

En las tablas I y II se exponen las características basales de la población. Como puede apreciarse en la tabla I, la edad media y los factores de riesgo de los pacientes con EAC no mostró diferencias significativas con los sujetos controles.

\begin{tabular}{|c|c|c|c|}
\hline \multicolumn{4}{|c|}{ TABLA I } \\
\hline \multicolumn{4}{|c|}{$\begin{array}{c}\text { CARACTERÍSTICAS BASALES DE LOS PACIENTES } \\
\text { CON ENFERM EDAD ARTERIAL CO RONARIA } \\
\text { (EAC) Y LOS CONTRO LES }\end{array}$} \\
\hline & $\mathrm{EAC}(\mathrm{n}=132)$ & Controles $(n=18)$ & $p$ \\
\hline Edad (años) & $61 \pm 10$ & $60 \pm 12$ & NS \\
\hline Tabaquismo (\%) & 27 & 22 & NS \\
\hline Hipertensión (\%) & 32 & 50 & NS \\
\hline Hipercolesterolemia (\%) & 42 & 33 & NS \\
\hline Hipertrigliceridemia (\%) & 12 & 16 & NS \\
\hline IIMC> $30(\%)$ & 26 & 27 & NS \\
\hline Sexo : Varones $(\%)$ & 76,51 & 38,8 & 0,001 \\
\hline Mujeres (\%) & 23,49 & 61,2 & 0,001 \\
\hline
\end{tabular}

IM C: Índice de masa corporal. NS: No nignificación estadística $(p \geq 0,05)$. 
TABLA ॥

PARÁM ETRO S CLÍNICOS DE LOS PACIENTES

CON EFERM EDAD ARTERIAL CORONARIA (EAC) Y LOS CONTRO LES

\begin{tabular}{lcc}
\hline & EAC ( $\mathrm{n}=132)$ & Controles $(\mathrm{n}=18)$ \\
\hline Angina estable (\%) & 47 & 0 \\
Angina inestable (\%) & 53 & 0 \\
Infarto antiguo (\%) & 20,1 & 0 \\
Insuficiencia mitral (\%) & 0 & 38,8 \\
Estenosis aórtica (\%) & 0 & 61,2 \\
Tratamiento (\%): & & \\
AASS & 98,5 & 0 \\
Diuréticos & 12,9 & 95 \\
IECA & 26,5 & 88,9 \\
Digital & 9,8 & 75,3 \\
Nitratos & 97,7 & 0 \\
B-bloqueantes & 95 & 0 \\
\hline
\end{tabular}

Con respecto al sexo si se observaron diferencias significativas entre pacientes con EAC y los controles. En lo que respecta al número de arterias coronarias principales afectadas, en los pacientes con EAC se apreciaba un $25,8 \% ; 34,8 \%$ y $39,4 \%$ con afección de 1, 2 y 3 vasos, respectivamente. En la tabla II se exponen los parámetros clínicos de la población de estudio, observándose los diferentes tratamientos y patología de los pacientes con EAC y los controles.

En la tabla III se exponen las concentraciones de HCY, creatinina, los diferentes parámetros lípidicos, fibrinógeno, glucosa, vitamina $B_{6}$, vitamina $B_{12} Y$ ácido fólico de los pacientes con EAC comparado con los controles, no mostrando diferencias significativas. La concentración media de los niveles de HCY en plasma para las mujeres con EAC comparado con sus controles fue de $11,96 \mu \mathrm{mol} / \mathrm{L}$ y $14,91 \mu \mathrm{mol} / \mathrm{L}$, respectivamente. Asimismo para los hombres con EAC comparado con sus controles fue de $12,40 \mu \mathrm{mol} / \mathrm{L}$ y 13,40 $\mu \mathrm{mol} / \mathrm{L}$, respectivamente; no existiendo entre ambos sexos diferencias significativas. En la distribución entre niveles de HCY por quintiles entre casos y controles no se encontró diferencias estadísticamente significativas $(p=0,57)$, por lo tanto la cantidad de HCY en plasma distribuida por quintiles no parece estar asociada a la producción de EAC.

En el análisis de regresión logística se incluyeron la hipertensión arterial, hipercolesterolemia, tabaquismo y los quintiles 2, 3, 4 y 5 de HCY, no existiendo una asociación significativa entre el nivel de HCY y el riesgo de EAC (Tabla IV).

\section{DISCUSIÓN}

En nuestro estudio, no se observaron diferencias significativas entre los niveles de HCY de los pacientes y controles. Los resultados de este estudio difieren de los obtenidos en numerosos estudios retrospectivos de casos y controles en los que se demostró que las concentraciones de HCY eran superiores en los pacientes con infarto agudo de miocardio (IAM) y EAC que en los sujetos de control (13).

Sin embargo hay algunas posibles explicaciones para las diferencias entre los resultados observados en el estudio y los observados en estudios retrospectivos de casos y controles.
TABLA III

PARÁM ETROS CLÍNICOS DE LOS PACIENTES

CON EFERM EDAD ARTERIAL CORO NARIA (EAC) Y LO S CONTRO LES

\begin{tabular}{|c|c|c|c|}
\hline & $E A C(n=132)$ & Controles $(n=18)$ & $P$ \\
\hline $\mathrm{HCY}(\mu \mathrm{mol} / \mathrm{l})$ & $12,30 \pm 8,78$ & $14,32 \pm 10,50$ & NS \\
\hline Colesterol total $(\mathrm{mmol} / \mathrm{l})$ & $5,89 \pm 1,28$ & $5,69 \pm 1,28$ & NS \\
\hline $\mathrm{LdL}$ colesterol (mmol/l) & $3,77 \pm 1,02$ & $3,64 \pm 1,18$ & NS \\
\hline HdL colesterol (mmol/l) & $1,08 \pm 0,28$ & $1,12 \pm 0,26$ & NS \\
\hline Triglicéridos (mmol/l) & $2,25 \pm 1,32$ & $2,01 \pm 1,06$ & NS \\
\hline Fibrinógeno (mmol/l) & $1,35 \pm 0,38$ & $1,34 \pm 0,35$ & NS \\
\hline Glucosa (mmol/l) & $5,35 \pm 0,72$ & $5,19 \pm 0,64$ & NS \\
\hline Vitamina $\mathrm{B}_{6}(\mathrm{nmol} / \mathrm{l})$ & $39,39 \pm 32,84$ & $41,01 \pm 26,28$ & NS \\
\hline Vitamina $B_{12}(p m o l / l)$ & $498,33 \pm 275,28$ & $516,97 \pm 192,66$ & NS \\
\hline Acido fólico (nmol/l) & $4,62 \pm 1,97$ & $4,55 \pm 1,72$ & NS \\
\hline Creatinina $(\mu \mathrm{mol} / \mathrm{l})$ & $82,22 \pm 18,56$ & $75,14 \pm 17,68$ & NS \\
\hline
\end{tabular}

Valores expresados como media $\pm \mathrm{DE}$; HCY: Homocísteina; NS: No significación estadística ( $p \geq 0,05)$.

TABLA IV

ANÁLISIS DE REGRESIÓN LO GÍSTICA

DE LOS FACTO RES DE RIESGO

\begin{tabular}{lccc}
\hline & ODDS RATIO & IC $(95 \%)$ & P \\
\hline Hipertensión arterial & 1,92 & $0,63-5,83$ & NS \\
Hipercolesterolemia & 0,69 & $0,20-2,30$ & NS \\
Tabaquismo & 0,49 & $0,13-1,76$ & NS \\
Quintil 2 de HCY & 0,60 & $0,11-3,09$ & NS \\
Quintil 3 de HCY & 0,47 & $0,07-2,92$ & NS \\
Quintil 4 de HCY & 0,67 & $0,13-3,51$ & NS \\
Quintil 5 de HCY & 1,67 & $0,39-7,03$ & NS \\
\hline
\end{tabular}

HCY: Homocisteína. Intervalo de confianza; IC: Intervalo de confianza; NS: No significación estadística $(p \geq 0,05)$.

En primer lugar es poco probable que el responsable de la incapacidad de encontrar una asociación entre la EAC y los niveles de HCY sea un efecto de la variación dietética de la HCY a corto plazo. El estudio realizado por Guttormsen et al (14) demostraron que después de la cena los niveles plasmáticos de HCY aumentaban un 13\%. Todos los participantes de este estudio estaban en ayunas antes de realizarse la extracción de sangre. No obstante sigue siendo posible una variación dietética, incluida la utilización de suplementos, que podrían haber disminuido los niveles de HCY entre el momento de la extracción de la muestra y el episodio cardiovascular.

En segundo lugar se ha indicado la posibilidad de que una prueba de carga con metionina mejoraría la capacidad de discriminar el riesgo de EAC con respecto a la medición de HCY en ayunas (15). Se ha publicado que los niveles delta de HCY postcarga de metionina se redujeron el $22 \%$, en promedio, con suplementos de vitamina $\mathrm{B}_{6}(50 \mathrm{mg} /$ día) (16), pero no de ácido fólico de hasta $5 \mathrm{mg} /$ día. Por ende, a veces no es posible normalizar la respuesta a la carga de metionina en todos los casos, lo que unido a la ausencia de evidencia del beneficio de 
una menor respuesta, hace que el valor clínico de esta prueba continúe siendo incierto (17).

En tercer lugar, es posible que las asociaciones observadas en los estudios de casos y controles retrospectivos puedan atribuirse a aumentos de las concentraciones de HCY que aparecen a raíz de un IAM. En el estudio realizado por Egerton et al (18) midieron los valores de HCY en sangre en el momento del IAM y hasta 180 días después del infarto. Las concentraciones de HCY fueron de 12,9 $\pm 0,9 \mu \mathrm{mol} / \mathrm{L}$ el día del infarto $\mathrm{y}$ del $15,3 \pm 1,1 \mu \mathrm{mol} / \mathrm{L}(\mathrm{p}=0,05) 180$ días después del infarto. En nuestro estudio la determinación de HCY en pacientes afectos de EAC no se realizó a aquellos con IAM, sino a pacientes afectos de sindrome anginoso y demostración angiográfica de lesiones ateromatosas.

En cuarto lugar un control inadecuado de los factores de riesgo cardiovasculares en los análisis estadísticos (factores de confusión), podría influir en la interpretación de las diferencias de los valores de HCY entre los casos y los controles. En el presente estudio ninguno de los factores de riesgo cardiovasculares constituyeron variables y/o factores de confusión.

En los estudios epidemiológicos en los que se demuestra la existencia de una correlación significativa entre los niveles de HCY y las enfermedades cardiovasculares tienden a informar de que las concentraciones de $\mathrm{HCY}$ en los pacientes son de $\approx 11 \mathrm{a}$ $16 \mu \mathrm{mol} / \mathrm{l}$, solo $3 \mu \mathrm{mol} / \mathrm{l}$ mayores que las del grupo control correspondiente (19). En el presente estudio las concentraciones de HCY son similares a los observados en otros estudios (20-21).

Es posible que las asociaciones encontradas entre los estudios retrospectivos con casos y controles puedan atribuirse a elevaciones de las concentraciones de HCY producidas tras un IM u otras enfermedades vasculares agudas. De esta forma, el hecho de encontrar concentraciones de HCY superiores en los pacientes caso que en los sujetos de control podría deberse a la enfermedad (IM) en lugar de una causa de IM o de enfermedad vascular.
En los últimos años han ido apareciendo los resultados de diferentes estudios prospectivos (22-25) que han investigado el papel del aumento de la HCY en el riesgo cardiovascular de muestras de la población general. El riesgo relativo conferido por la hiperhomocisteinemia era sustancialmente menor al calculado a partir de estudios de casos y controles. Ello, junto a los resultados negativos de los estudios sobre el polimorfismo T677 de la metilentetrahidrofolato reductasa (26-28), ha generado serias dudas sobre si la hiperhomocisteinemia moderada es un factor de riesgo cardiovascular en la población general. Lamentablemente, aún no hay resultados de estudios a largo plazo que demuestren un efecto beneficioso de la reducción de las concentraciones de HCY sobre la incidencia de eventos cardiovasculares y mortalidad (29) Por ello, se ha dividido a los factores de riesgo (30) para EAC en tres tipos: Factores de riesgo principales independientes (fumador, hipertensión arterial, colesterol total y LdL elevado, HdL disminuido, diabetes mellitus y edad avanzada); Factores de riesgo predisponentes (obesidad, inactividad física, antecedentes familiares para enfermedad coronaria, características étnicas y factores psicosociales) y Factores de riesgo condicionales (hipertrigliceridemia, partículas LdL pequeñas, HCY, Lpa elevada y proteína C-reactiva).

En conclusión, a la vista de nuestros resultados se puede interpretar que no se sustenta la hipótesis de que el aumento de los niveles séricos de HCY sea factor de riesgo independiente para EAC. Aunque se trata de un estudio de diseño de casos y controles, donde el número de sujetos controles es bajo, la variabilidad que muestra el grupo de casos es similar al de los controles, indicando una tendencia a corroborar que con este grupo podría ser suficiente para las afirmaciones realizadas. No obstante, creemos que sea necesario estudios controlados de intervención clínica para aclarar la relación entre la HCY y la enfermedad cardiovascular.

\section{Bibliografía}

1. Cordoba Porras A, Blanco Vaca F, González Sastre F. Hiperhomocisteinemia, un nuevo marcador de riesgo vascular: territorios vasculares afectados, papel en la patogénesis de la arteriosclerosis y la trombosis y tratamiento. Med Clin (Barc) 1997; 109: 715-725.

2. Hankey GJ, Eikelboom JW. Homocysteine and vascular disease. Lancet 1999; 354: 407-13.

3. Stampfer MJ, Malinow MR, Willet WC, Newcomer LM, Upson B, Ullman D et al. A prospective study of plasma homocysteine and risk of miocardial infarction in US physicians. JAMA 1992; 268: 877-881

4. Chasan-Taber L, Selhub J, Rosenberg IH, Malinow R, Terry P, Tishler $\mathrm{PV}$ et al. A prospective study of folate and vitamin B6 and risk of miocardial infarction in US physicians. J Am Coll Nutr 1996; 15: 136-143.

5. Verhoef P, Hennekens CH, Allen RH, Stabler SP, Willett WC, Stampfer MJ. Plasma total homocysteine and risk of angina pectoris with subsequent coronary artery bypass surgery. Am J Cardiol 1997; 79: 799-801.

6. Alfthan G, Pekkanen J, Jauhiainen M, Pitkaniemi J, Karvonen M, Tuomilehto J et al. Relation of serum homocysteine and lipoprotein (a) concentrations to atherosclerotic disease in a prospective finish population based study. Atherosclerosis 1994; 106: 9-19.

7. Robinson K, Mayer EL, Miller DP, Green R, Van Lente F, Gupta A et al. Hyperhomocysteinemia and low pyridoxal phosphate. Common and independent reversible risk factors for coronary artery disease. Circulation 1995; 92: 2825-2830.

8. Dudman NP, Wilcken DE, Wang J, Lynch JF, Macey D, Lundberg PD. Methionine/homocysteine metabolism in premature vascular disease: its occurrence, cofactor therapy, and enzymology. Arterioscler Thromb Vasc Biol 1993; 12: 1253-60.

9. C Fernández-Miranda, J L Aranda, P Gómez González, P Díaz-Rubio, J Estenoz y A Gómez de la Cámara. La hiperhomocisteinemia es frecuente en pacientes con enfermedad coronaria. Estudio de 202 enfermos. Med Clin (Barc) 1999; 113: 407-410.

10. Frantzen F, Faaren AL, Alfheim I, Nordhei AK. Enzyme conversion immunoassay for determining total homocysteine in plasma or serum. Clin Chem 1998; 44: 311- 316.

11. Folsom AR, Nieto J, McGovern PG, Tsai MY, Malinow MR, Eckfeldt JH et al. Prospective study of coronary heart disease incidence in relation to fasting total homocysteine, related genetic polymorphisms, and B vitamins: the Atherosclerosis Risk in Communities (ARIC) study. Circulation 1998; 98: 204-10.

12. Borenstein M, Rothstein H, Cohen J. 1997. Samplepower 1.2, Illinois.

13. Boushey CJ, Beresford SAA, Omenn GS, Motulsky AG. A quantitative assessment of plasma homocysteine as a risk factor for vascular disease: probable benefits of increasing folic acid intakes. JAMA 1995; 274: 1049-1057.

14. Guttormsen AB, Scheede J, Fiskerstrand T, Ueland PM, Refsum HM. Plasma concentrations of homocysteine and other aminothiol compounds are related to food intake in healthy human subjects. J Nutr 1994; 124: 1934-41. 
15. Bostom AG, Jacques PF, Nadeau MR, Williams RR, Ellison RC, Selhub J. Post-methionine load hyperhomocysteinemia in persons with normal fasting total plasma homocysteine: initial results from the NHLBI Family Heart Study. Atherosclerosis 1995; 116: 147-151.

16. Bostom AG, Gohh RY, Beaulieu AJ, Nadeau MR, Hume AL, Jacques $\mathrm{PF}$ et al. Treatment of hyperhomocisteinemia in renal transplant recipients: a randomized, placebo-controlled trial. Ann Inten Med 1997; 127: 1089-1092.

17. Malinow MR, Bostom AG, Krauss RM. Homocyst(e)ine, Diet, and Cardiovascular Diseases. Circulation 1999; 99: 178-82.

18. Egerton W, Silberberg J, Crooks R, Ray C, Dudman N. Serial measures of plasma homocysteine after acute myocardial infarction. Am J Cardiol 1996; 77: 759-761.

19. Kuller LH, Evans RW. Homocysteine, vitamins, and cardiovascular disease. Circulation 1998; 98: 196-199.

20. Evans RW, Shaten J, Hempel JD, Cutler JA, Kuller LH for the MRFIT research Group. Homocyst(e)ine and risk of cardiovascular disease in the Multiple Risk Factor Intervention Trial. Arterioscler Thromb Vasc Biol 1997; 17: 1947-53.

21. Pery IJ, Refsum H, Morris RW, Ebrahim SB, Ueland PM, Shaper AG. Prospective study of serum total homocysteine concentration and risk of stroke in middle-aged British men. Lancet 1995; 346: 1395-98.

22. Wald NJ, Watt HC, Law MR, Weir DG, McPartlin J, Scott JM. Homocysteine and ischemic heart disease: results of a prospective study with implications regarding prevention. Arch Interm Med 1998; 158: 862-67.
23. Arnesen E, Refsum H, Bonaa KJ, Ueland PM, Forde OH, Nordrehaug JE. Serum total homocysteine and coronary heart disease. Int J Epidemiol 1995; 24: 704-09.

24. Nygard O, Nordrehaug JE, Refsum H, Ueland PM, Farstad M, Vollset SE. Plasma homocysteine levels and mortality in patients with coronary artery disease. N Engl J Med 1997; 337: 230-36.

25. Verhoef P, Hennekens CH, Malinow MR, Kok FJ, Willett WC, Stampfer MJ. A prospective study of plasma homocysteine and risk of ischemic stroke. Stroke 1994; 25: 1924-30.

26. Hess DL et al. Myocardial infarction in young women in relation to plasma homocysteine, folate, and a common variant in the methylenetetrahydrofolate reductase gene. Circulation 1997; 96: 412-417.

27. Methylenetetrahydrofolate reductase polymorphism, plasma folate, homocysteine, and risk of myocardial infarction in US physicians. Circulation 1996; 94: 2410-2416.

28. Wilcken DE, Wang XL, Sim AS, McCredie RM. Distribution in healthy and coronary populations of methylenetetrahydrofolate reductase (MTHFR) C677T mutation. Arterioscler Thromb Vasc Biol 1996; 16: 878-882.

29. Bunout D, Escobar Edgardo. Prevención de enfermedades cardiovasculares: ¿deben aplicarse los mismos criterios en América Latina que en Europa y Norteamérica?. Rev Esp Cardiol 2000; 53: 889-895.

30. Grundy SM, Pasternak R, Greenland P, Smith JR S, Fuster V. Assessment of Cardiovascular Risk by Use of Multiple-Risk-Factor Assessment Equations. A Statement for Healthcare Professionals From the American Heart Association and the American College of Cardiology. J Am Coll Cardiol 1999; 34: 1348-59. 